\title{
Cause-Specific Risk Factors of Death in Individuals with Diabetes: A Competing Risks Modeling
}

\author{
Marjan Mansourian ${ }^{1}$, Sahar Sadeghpour ${ }^{1}$, Ashraf Aminorroaya ${ }^{2}$, Masoud Amini ${ }^{2}$ and Tohid \\ Jafari-Koshki ${ }^{3,4, *}$ \\ ${ }^{1}$ Department of Epidemiology and Biostatistics, School of Public Health, Isfahan University of Medical Sciences, Isfahan, Iran \\ ${ }^{2}$ Isfahan Endocrine and Metabolism Research Centre, Isfahan University of Medical Sciences, Isfahan, Iran \\ ${ }^{3}$ Medical Education Research Center, Health Management and Safety Promotion Research Institute, Tabriz University of Medical Sciences, Tabriz, Iran \\ ${ }^{4}$ Department of Statistics and Epidemiology, Faculty of Health, Tabriz University of Medical Sciences, Tabriz, Iran \\ "Corresponding author: Medical Education Research Center, Health Management and Safety Promotion Research Institute, Tabriz University of Medical Sciences, Postal Code: \\ 5165665931, Tabriz, Iran. Tel: +98-9144926048, Email: tjkoshki@gmail.com
}

Received 2018 April 15; Revised 2019 April 16; Accepted 2019 May 18.

\section{Abstract}

Background: Diabetes is on the rise worldwide.

Objectives: This study aimed to evaluate the risk factors of various causes of death in people with type 2 diabetes (T2D).

Methods: In this cohort study on 2638 people with T2D, we applied cause-specific and sub-distribution hazards models to assess the impact of various factors on the risk of death. Moreover, we plotted a cumulative incidence curve to summarize cumulative failure rates over time.

Results: About 75\% of individuals with T2D died from cardiovascular disease (CVD) and cerebrovascular accidents (CVA). Death from CVD was associated with the increased risk of hypertension (hazard ratio $(\mathrm{HR})=1.83,95 \% \mathrm{CI}: 1.37-2.46)$, hypercholesterolemia $(\mathrm{HR}=$ 1.58, 95\% CI: 1.17 - 2.14), and diabetes duration. The risk of death from CVA was related to hypertension (HR $=2.76,95 \% \mathrm{CI}: 1.67-4.55)$ and hyperglycemia ( $\mathrm{HR}=4.34,95 \% \mathrm{CI}: 1.75-10.79)$. The CVA risk in patients with diabetes duration of $10-20$ years was higher than the risk in patients with diabetes duration $>20$ years (diabetes duration of $\leq 10$ years as the reference category). Diabetes duration of longer than 20 years was associated with a higher risk of death from cancer (HR=2.65, 95\% CI:1.05 - 6.68). The risk of death from foot infection and diabetic nephropathy increased in patients with longer diabetes duration after adjustment for sex, age, and body mass index.

Conclusions: Regardless of the cause, death rates in people with T2D increase over time and risk factors have different impacts on death from each cause. This should be acknowledged in risk management in individuals with T2D.

Keywords: Diabetes, Mortality, CVD, CVA, Cancer, Competing Risks

\section{Background}

Individuals with type 2 diabetes (T2D) are more vulnerable to both short- and long-term complications often leading to premature death and reduced life expectancy $(1$, 2 ). The risk of cardiovascular disease (CVD) and stroke in people with T2D is 2 - 4 and 1.5 - 5 times that of the general population, respectively. CVD in the T2D population accounts for almost 70\% of deaths and stroke has higher severity and mortality among this population (3-5). Almost $20 \%$ of deaths in patients with T2D are attributed to cerebrovascular accidents (CVA) (6). There is increasing biological and epidemiological evidence of the association between diabetes (mainly type 2) and the increased risk of cancer in advanced stages with high levels of mortality (79). Foot infections and ulcers are important causes of hos- pitalization and the leading causes of morbidity in T2D (10). The prevalence of renal disorders in individuals with T2D is estimated to be 17 times that in the general population. These include microvascular complications such as diabetic nephropathy that is the main cause of the endstage renal disease (ESRD) worldwide $(5,11)$.

Generally, patients with T2D may experience different events and the corresponding risk factors among them are usually evaluated using logistic regression. However, this approach overlooks time to occurrence of the event of interest. From this viewpoint, time-to-event or survival analysis methods are preferred. However, when more than a single event is evaluated, simple methods such as Cox regression could give inaccurate estimates, as they ignore associations between the events that idiomatically compete 
against one another to occur earlier. In an effort to settle this issue, competing risks models have been suggested.

Despite the popularity of competing risks modeling in various disciplines of medicine, studies using this approach in the diabetes context are not frequent (12-14). In a recent study, the risk of death from CVD, cancer, and other (non-cardiovascular-non-cancer) causes has been evaluated without evaluating risk factors of each death type (2). Nevertheless, there are studies in patients with T2D that examined risk factors for a particular cause $(15,16)$.

\section{Objectives}

The current study aimed to examine the cause-specific risk factors for each cause of death (CVD, CVA, cancer, and foot infection/diabetic nephropathy) in T2D using competing risks analysis.

\section{Methods}

\subsection{Study Population}

We retrieved the data of 2638 people with T2D (1110 men and 1528 women) from the database of the Isfahan Endocrine and Metabolism Research Center from 1992 to 2004 with a median follow-up of 60 months. All patients aged $\geq 35$ were included in the analysis. Type 1 diabetes, death from causes other than those under study, and missing data were the criteria for exclusion from the analysis.

Of the 2638 people with T2D, 395 (15\%) suffered from various risk events including death from CVD, CVA, cancer, and foot infections/diabetic nephropathy. There were 215 patients $(8.2 \%)$ who died of myocardial infarction, 89 (3.4\%) of stroke, 54 (2\%) of cancer, and 37 (1.4\%) of foot infections/diabetic nephropathy; thus, 2243 (85\%) patients did not experience any of the final points considered as censored in this study.

All cases of coronary heart disease, congestive heart failure, rheumatic heart disease, cardiomyopathy, and other heart diseases were considered as CVD. Ischemic stroke and hemorrhagic stroke were included in the CVA definition. Individuals with a history of any events under study at the baseline were excluded from the study.

\subsection{Measurements and Outcomes}

Demographic information and duration of diabetes were recorded by a trained interviewer using a standardized questionnaire. An inflexible bar was used to measure the height of participants standing straight against the wall. A digital scale was used to measure weight rounded to the nearest $100 \mathrm{~g}$. Measurements were done in light clothing taking socks and shoes off. Blood pressure (BP) was measured after 15-minutes rest in the sitting position with appropriately sized cuffs using a standardized mercury sphygmomanometer on the right arm at two timepoints of the 5-minutes interval. The mean of these measures was recorded as BP if the differences between the two measures of systolic and diastolic BP were less than 10. Otherwise, a third measurement was done and the average was recorded. Patients with a history of antihypertensive drug use, SBP $\geq 140 \mathrm{mmHg}$, or DBP $\geq 90 \mathrm{mmHg}$ were considered hypertensive $(17,18)$.

Fasting blood sugar (FBS), triglyceride (TG), and cholesterol levels were evaluated using blood tests. Those with FBS $\geq 126 \mathrm{mg} / \mathrm{dL}$ were considered hyperglycemic. TG and cholesterol levels were categorized into two groups in 200 and $240 \mathrm{mg} / \mathrm{dL}$, respectively. Most T2D patients are exposed to various risks after the age of 65 . Therefore, we classified patients into two groups of $\leq 65$ and $>65$ years in the analysis. Detailed information on measurements and procedures is available elsewhere (15).

The composite outcome was the time from diabetes diagnosis to death from CVD, CVA, cancer, or foot infection/diabetic nephropathy. Patients who lost the follow-up or experienced none of these endpoints in the study period were considered as censored in the analysis. Hence, the survival time was the time from diagnosis of diabetes to experiencing the event, last visit, or end of the study.

\subsection{Statistical Analysis}

We used two main approaches introduced in the context of competing risks models. Each method answers a different research question. The following is a brief review of these methods. More details can be found elsewhere (19, 20).

The first model for analyzing competing risks data is the cause-specific hazard ( $\mathrm{CSH}$ ) model that is a generalization of the traditional Cox model to compensate for the overestimation bias (19). In this model, the effect of covariates on the risk of experiencing each of competing events at any specific time is evaluated over those subjects who have experienced none of the events up to that time. That is, the CSH model gives an instant rate of occurrence of each event in subjects who have not yet experienced either event. The interpretation of associations is through the well-known hazard ratio (HR) in a similar way to the Cox regression. The second method is the subdistribution hazards (SDH) model introduced by Fine and Gray in 1999 (21). In this approach, instead of the modeling hazard function, cumulative incidence function (CIF) is modeled. Thus, the SDH model transfers a different concept. At any time, the effect of covariates on the risk of experiencing each of competing events is evaluated over all subjects who have not have that event until that time. Here, at any time, the risk 
set includes all subjects in the study except for those who have experienced the event under consideration.

The CSH and SDH models use different methods and answer different types of questions, as well. In brief, the SDH model is used when the researcher aims to assess the overall impact of a covariate on the incidence of each event, i.e. the estimation of actual risks and prognosis, or risk prediction for a specific person. The CSH model is more concerned with studying the etiology of diseases (19). Nevertheless, less computational demand and simple interpretations are the merits of the CSH model. The SDH model not only informs patients about the risks they face in certain situations, but also can guide clinicians in assigning a specific treatment regimen to a patient (22).

Cumulative incidence of an event is often of interest and is frequently reported in medical research. The graphical display of the CIF (i.e., failure probabilities) over time is intuitive and appealing. In the presence of competing risks, a class of tests has been proposed in the literature for comparing the cumulative incidence curves of a particular type of failure among different groups (23).

We included the following predictors altogether in one-step analysis: duration of diabetes, blood pressure, hyperglycemia, triglyceride, and hypercholesterolemia. The results were adjusted for age, sex, and BMI.

Statistical analysis was carried out using R package cmprsk (http://www.r-project.org). Descriptive statistics were presented as No. (\%) and mean \pm SD. The statistical significance level was set at 0.05 .

\section{Results}

The baseline characteristics of the total sample of 2638 subjects included in the final analysis are shown in Table 1. Briefly, $57.9 \%$ of the patients were female and the mean age of the patients was $55.16 \pm 9.86$ years $(\min =35$, $\max =92)$. Of the total sample, 215 (8.2\%) patients died from CVD, 89 (3.4\%) from CVA, 54 (2\%) from cancer, and 37 (1.4\%) from foot infection/diabetic nephropathy that accounted for $54.4 \%$, $22.5 \%, 13.7 \%$, and $9.4 \%$ of the total deaths in this population, respectively.

The HR and corresponding 95\% confidence intervals (CI) after adjusting for sex, age, and BMI are shown in Table 2. The risk of death from CVD increased with hypertension $(\mathrm{HR}=1.83$, 95\% CI: $1.37-2.46)$, hypercholesterolemia $(\mathrm{HR}=$ 1.58, 95\% CI: $1.17-2.14)$, and diabetes duration. Hypertension ( $\mathrm{HR}=2.76,95 \% \mathrm{CI}: 1.67-4.55)$ and hyperglycemia (HR $=4.34$, 95\% CI:1.75 - 10.79) were associated with higher risks of death from CVA and the risk in patients with diabetes duration of 10 - 20 years was higher than the risk of other patients. Diabetes duration of longer than 20 years was associated with a higher risk of death from cancer $(\mathrm{HR}=2.65$,
95\% CI: 1.05 - 6.68). In addition, patients with diabetes duration of 10-20 years were at higher risk of death from foot infection/diabetic nephropathy than the patients of other ages $(\mathrm{HR}=2.20$, 95\% CI: 1.07 - 4.50). Almost similar results were seen in both analyses with stronger associations in the CSH models as expected. However, the factors associated with the last two death causes were found to be significant only in the CSH model.

According to the estimate of the cumulative incidence for competing events at various time points shown in Table 3 , the probability of death from CVD, CVA, cancer, and foot infection/diabetic nephropathy was respectively $8.4 \%, 3.3 \%$, $2.0 \%$, and $1.4 \%$ after 120 months and $8.7 \%, 3.6 \%, 2.1 \%$, and $1.4 \%$ after 216 months.

Figure 1 summarizes the cumulative incidence estimates for all the outcomes taking competing risks into accounts. The probability of death due to CVD, CVA, cancer, and foot infection/diabetic nephropathy was almost the same until the 24th month. However, after month 24, the risk of death from CVD was relatively higher than the risk from other events. In other words, the risk of death from any cause increases over time and patients who develop CVD are at higher risk of death, especially after 24 months.

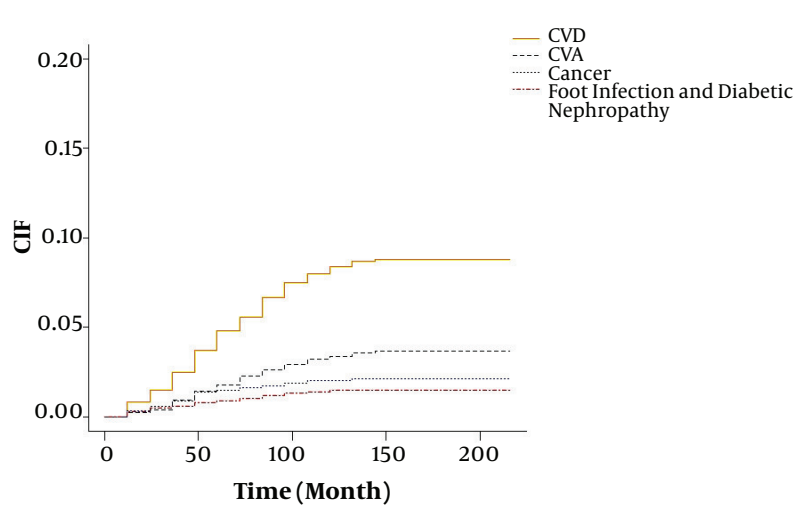

Figure 1. Competing risks cumulative incidence curves for all the outcomes. Abbreviations: CVD, cardiovascular disease; CVA, cerebrovascular accident; CIF, cumulative incidence function.

\section{Discussion}

In this study, we used CSH and SDH models for modeling competing risks of death and related risk factors in patients with T2D. After adjusting for age, sex, and BMI, hypercholesterolemia and diabetes duration of longer than 10 years were found to be associated with higher risks of death from CVD. Moreover, hypertension, higher FBS levels, and diabetes duration of 10 - 20 years increased the risk 


\begin{tabular}{|c|c|c|c|c|c|}
\hline Variable & Total $(\mathrm{N}=\mathbf{2 6 3 8})$ & $\operatorname{CVD}(\mathrm{N}=215)$ & $\operatorname{CVA}(\mathbf{N}=\mathbf{8 9})$ & Cancer $(N=54)$ & Foot Infection/Diabetic Nephropathy $(\mathrm{N}=37)$ \\
\hline \multicolumn{6}{|l|}{ Gender } \\
\hline Female & $1528(57.9)$ & $81(37.7)$ & $37(41.6)$ & $22(40.7)$ & $13(35.1)$ \\
\hline Male & $1110(42.1)$ & $134(62.3)$ & $52(58.4)$ & $32(59.3)$ & $24(64.9)$ \\
\hline \multicolumn{6}{|l|}{ Age, $y$} \\
\hline$\leq 65$ & $2272(86.1)$ & $159(74.0)$ & $56(62.9)$ & $40(74.1)$ & $28(75.7)$ \\
\hline$>65$ & $366(13.9)$ & $56(26.0)$ & $33(37.1)$ & $14(25.9)$ & $9(24.3)$ \\
\hline \multicolumn{6}{|l|}{ BMI, kg/m² } \\
\hline$\leq 25$ & $718(27.2)$ & $88(40.9)$ & $38(42.7)$ & $20(37.0)$ & $18(48.6)$ \\
\hline$>25$ & $1920(72.8)$ & $127(59.1)$ & $51(57.3)$ & $34(63.0)$ & $19(51.4)$ \\
\hline \multicolumn{6}{|c|}{ Diabetes duration, $y$} \\
\hline$\leq 10$ & $2072(78.5)$ & $136(63.3)$ & $52(58.4)$ & $38(70.4)$ & $20(54.1)$ \\
\hline $10-20$ & $474(18.0)$ & $60(27.9)$ & $32(36.0)$ & $10(18.5)$ & $13(35.1)$ \\
\hline$>20$ & $92(3.5)$ & $19(8.8)$ & $5(5.6)$ & 6(11.1) & $4(10.8)$ \\
\hline \multicolumn{6}{|l|}{ Hypertension } \\
\hline Yes & $1319(50.0)$ & $145(67.4)$ & $68(76.4)$ & $33(61.1)$ & $25(67.6)$ \\
\hline No & $1319(50.0)$ & $70(32.6)$ & $21(23.6)$ & $21(38.9)$ & $12(32.4)$ \\
\hline \multicolumn{6}{|l|}{ FBS, mg/dL } \\
\hline$\leq 126$ & $600(22.7)$ & $33(15.3)$ & $5(5.6)$ & $8(14.8)$ & $2(5.4)$ \\
\hline$>126$ & $2038(77.3)$ & $182(84.7)$ & $84(94.4)$ & $46(85.2)$ & $35(94.6)$ \\
\hline \multicolumn{6}{|c|}{ Triglyceride, mg/dL } \\
\hline$\leq 200$ & $1677(63.6)$ & $121(56.3)$ & $51(57.3)$ & $43(79.6)$ & $17(45.9)$ \\
\hline$>200$ & $961(36.4)$ & $94(43.7)$ & $38(42.7)$ & $11(20.4)$ & $20(54.1)$ \\
\hline \multicolumn{6}{|c|}{ Cholesterol, mg/dL } \\
\hline$\leq 240$ & $2000(75.8)$ & $141(65.6)$ & $65(73.0)$ & $46(85.2)$ & $22(59.5)$ \\
\hline$>240$ & $638(24.2)$ & $74(34.4)$ & $24(27.0)$ & $8(14.8)$ & $15(40.5)$ \\
\hline
\end{tabular}

Abbreviations: BMI, body mass index; CVA, cerebrovascular accident; CVD, cardiovascular disease; FBS, fasting blood sugar.

${ }^{\mathrm{a}}$ Values are expressed as No. (\%).

of death from CVA. However, the impact of diabetes duration on CVA death risk was not significant for durations of longer than 20 years. This may be due to the small number of patients in this category. Meanwhile, other unknown biological processes may have played a role.

Finally, long diabetes duration significantly affected death from foot infection/diabetic nephropathy. As expected, the HRs estimated by the CSH model were higher than those estimated by the SDH model. The negligence of the association inherently existing between competing events may have influenced the results and interpretation of the prognostic effects of different events.

Our results are in concordance with the findings from previous studies. We found a direct association between hypertension and hypercholesterolemia and the risk of death due to CVD in patients with T2D. It has been reported that higher blood pressure is a strong stimulus for CVD in people with T2D (24-26). Among various risk factors for CVD, the relationship between hypercholesterolemia and CVD has long been recognized $(27,28)$. An epidemiologic survey demonstrated that elevated levels of total cholesterol and LDL-C were associated with increased risk of coronary heart disease (29). A Finnish study showed that the mortality rate from CVD among people with high levels of cholesterol was five times that in the general population and the reduction of serum cholesterol levels up to $10 \%$ could reduce mortality due to CVD up to $30 \%$ (30).

The duration of diabetes also increased the risk of death from CVD in patients with T2D. Moreover, T2D was associated with elevated total and coronary heart disease mortality and the longer duration of diabetes was reported to be a strong predictor of death among these patients (31, 


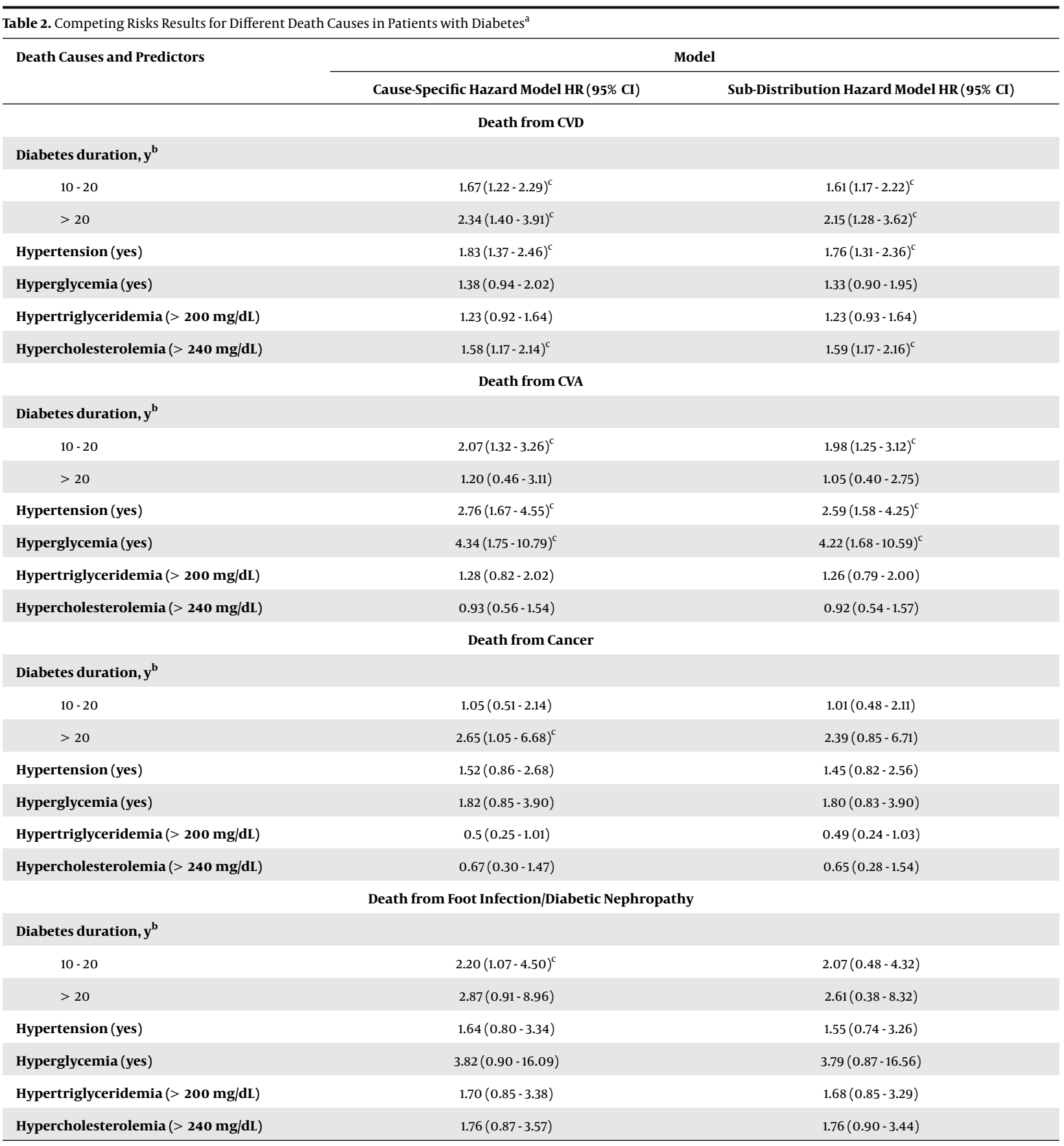

Abbreviations: CVA, cerebrovascular accident; CVD, cardiovascular disease; HR, hazard ratio.

${ }^{a}$ Results are adjusted for age, sex, and BMI.

${ }^{\mathrm{b}}$ Diabetes duration of $\leq 10$ years as the reference category

${ }^{\mathrm{c}}$ Significant factors.

32).

It is claimed that CVD is the main cause of death in patients with T2D and the rates of mortality from natural causes rise when the duration of diabetes increases (33).

We found a significant association between blood pres- sure and fasting blood sugar and the risk of death due to stroke in T2D patients. It has been suggested that these factors increase the risk of stroke even after adjustment for other variables $(34,35)$. Moreover, our results indicated that the duration of diabetes was positively correlated with 


\begin{tabular}{|c|c|c|c|c|}
\hline \multirow[b]{2}{*}{ Time, mo } & \multicolumn{4}{|c|}{ Type of Event } \\
\hline & Cardiovascular Disease & Cerebrovascular Accident & Cancer & Foot Infection/Diabetic Nephropathy \\
\hline 24 & $0.014(5.55 e-06)$ & $0.003(1.44 \mathrm{e}-06)$ & $0.006(2.30 \mathrm{e}-06)$ & $0.004(1.86 e-06)$ \\
\hline 48 & $0.037(1.36 \mathrm{e}-05)$ & $0.014(5.35 e-06)$ & $0.013(5.19 \mathrm{e}-06)$ & $0.008(3.03 e-06)$ \\
\hline 72 & $0.055(2.08 \mathrm{e}-05)$ & $0.022(8.80 e-06)$ & $0.016(6.24 \mathrm{e}-06)$ & $0.010(4.09 \mathrm{e}-06)$ \\
\hline 96 & $0.074(2.80 e-05)$ & $0.029(1.15 e-05)$ & $0.018(7.30 \mathrm{e}-06)$ & $0.013(5.34 \mathrm{e}-06)$ \\
\hline 120 & $0.084(3.15 e-05)$ & $0.033(1.33 \mathrm{e}-05)$ & $0.020(8.03 e-06)$ & $0.014(5.91 e-06)$ \\
\hline 168 & $0.087(3.29 e-05)$ & $0.036(1.45 e-05)$ & $0.021(8.42 \mathrm{e}-06)$ & $0.014(5.91 e-06)$ \\
\hline 192 & $0.087(3.29 e-05)$ & $0.036(1.45 e-05)$ & $0.021(8.42 \mathrm{e}-06)$ & $0.014(5.91 \mathrm{e}-06)$ \\
\hline 216 & $0.087(3.29 \mathrm{e}-05)$ & $0.036(1.45 e-05)$ & $0.021(8.42 \mathrm{e}-06)$ & $0.014(5.91 e-06)$ \\
\hline
\end{tabular}

stroke. This is in accordance with the findings of previous research where the risk of stroke in T2D patients was reported to be three times that in the general population (36, 37).

The duration of diabetes has been declared to be one of the most important factors in the risk of cancer and death among T2D patients (38). Similarly, diabetes duration predicts the progression of nephropathy in these patients (39, 40). Diabetes duration could also predispose both men and women to diabetic foot ulcers $(41,42)$. It should be noted that, in general, the estimated covariate effects using the CSH and SDH models may be different (13).

Our study is not free of limitations. First, part of data, such as diabetes duration, relied on self-reports. Furthermore, the sample size was intrinsically low for some risk factors, e.g. long diabetes duration, and outcomes, e.g. death from foot infection/diabetic nephropathy. This led to the low power of the tests for these groups as, despite clinically large estimates of HRs, the associations were not statistically significant. Steady cumulative incidence after about 150 months could be the effect of low sample size, as well.

This study examined the impact of various risk factors on the risk of death from different events in individuals with T2D using competing risks analysis that is a better choice in the presence of multiple possible events. Studies in competing risks setting usually report the results of one analytic method. We implemented both $\mathrm{CSH}$ and $\mathrm{SDH}$ models to give information for target-specific interventions and attain the comprehensiveness of the study.

\subsection{Conclusions}

Regardless of the cause, death rates in individuals with T2D increase over time and risk factors have different impacts on death from each cause. This should be acknowledged in risk management in individuals with T2D.

\section{Footnotes}

Authors' Contribution: Marjan Mansourian and Ashraf Aminorroaya conceptualized the study and participated in discussion. Marjan Mansourian and Sahar Sadeghpour analyzed the data and prepared initial draft. Masoud Amini participated in data collection, literature review and draft preparation. Tohid Jafari-Koshki participated in study design and data analysis and revised the manuscript. All authors approved the final manuscript.

Conflicts of Interests: It is not declared by the authors.

Ethical Approval: The protocol of the IDPS was approved by the Ethics Committee of the Isfahan University of Medical Sciences.

Funding/Support: This study is supported financially by the Isfahan University of Medical Sciences.

Patient Consent: Informed consent was obtained from all individual participants included in the study.

\section{References}

1. Jafari-Koshki T, Mansourian M, Hosseini SM, Amini M. Association of waist and hip circumference and waist-hip ratio with type 2 diabetes risk in first-degree relatives.J Diabetes Complications. 2016;30(6):10505. doi: 10.1016/j.jdiacomp.2016.05.003. [PubMed: 27311785].

2. Baena-Diez JM, Penafiel J, Subirana I, Ramos R, Elosua R, Marin-Ibanez A, et al. Risk of cause-specific death in individuals with diabetes: A competing risks analysis. Diabetes Care. 2016;39(11):1987-95. doi: 10.2337/dc16-0614. [PubMed: 27493134].

3. Yamagishi S. Cardiovascular disease in recent onset diabetes mellitus. J Cardiol. 2011;57(3):257-62. doi: 10.1016/j.jjcc.2011.01.011. [PubMed: 21367583].

4. Gaede P, Vedel P, Larsen N, Jensen GV, Parving HH, Pedersen O. Multifactorial intervention and cardiovascular disease in patients with type 2 diabetes. N Engl J Med. 2003;348(5):383-93. doi: 10.1056/NEJMoa021778. [PubMed: 12556541].

5. Jafari-Koshki T, Hosseini SM, Arsang-Jang S, Amini M, Faghihimani E. Trends of diabetic nephropathy prevalence in Isfahan, Iran, during 1992-2010. J Res Med Sci. 2015;20(10):944-9. doi: 10.4103/17351995.172781. [PubMed: 26929758]. [PubMed Central: PMC4746867]. 
6. Phipps MS, Jastreboff AM, Furie K, Kernan WN. The diagnosis and management of cerebrovascular disease in diabetes. Curr Diab Rep. 2012;12(3):314-23. doi: 10.1007/s11892-012-0271-x. [PubMed: 22492061].

7. Giovannucci E, Harlan DM, Archer MC, Bergenstal RM, Gapstur SM, Habel LA, et al. Diabetes and cancer: A consensus report. CA CancerJ Clin. 2010;60(4):207-21. doi: 10.3322/caac.20078.

8. Griffiths RI, Danese MD, Gleeson ML, Valderas JM. Epidemiology and outcomes of previously undiagnosed diabetes in older women with breast cancer: An observational cohort study based on SEERMedicare. BMC Cancer. 2012;12:613. doi: 10.1186/1471-2407-12-613. [PubMed: 23259613]. [PubMed Central: PMC3575284].

9. Toriola AT, Stolzenberg-Solomon R, Dalidowitz L, Linehan D, Colditz G. Diabetes and pancreatic cancer survival: A prospective cohort-based study. Br J Cancer. 2014;111(1):181-5. doi: 10.1038/bjc.2014.224. [PubMed: 24786605]. [PubMed Central: PMC4090724].

10. Gadepalli R, Dhawan B, Sreenivas V, Kapil A, Ammini AC, Chaudhry R. A clinico-microbiological study of diabetic foot ulcers in an Indian tertiary care hospital. Diabetes Care. 2006;29(8):1727-32. doi: 10.2337/dc06-0116. [PubMed:16873771].

11. Liao EP. Management of type 2 diabetes: New and future developments in treatment. Am J Med. 2012;125(10):S2-3. doi: 10.1016/j.amjmed.2012.05.008. [PubMed: 22998892].

12. Toya K, Babazono T, Murata H, Hanai K, Uchigata Y. Association of serum bilirubin levels with mortality in patients with diabetes initiating chronic hemodialysis: A competing risks analysis of a single-center cohort. Renal Replacement Therapy. 2016;2(1). doi: 10.1186/s41100-016-0065-8.

13. Lim HJ, Zhang X, Dyck R, Osgood N. Methods of competing risks analysis of end-stage renal disease and mortality among people with diabetes. BMC Med Res Methodol. 2010;10:97. doi: 10.1186/1471-2288-10-97. [PubMed: 20964855]. [PubMed Central: PMC2988010].

14. Forsblom C, Harjutsalo V, Thorn LM, Waden J, Tolonen N, Saraheimo $\mathrm{M}$, et al. Competing-risk analysis of ESRD and death among patients with type 1 diabetes and macroalbuminuria. J Am Soc Nephrol. 2011;22(3):537-44. doi: 10.1681/ASN.2010020194. [PubMed: 21335512] [PubMed Central: PMC3060447].

15. Sadeghpour S, Faghihimani E, Hassanzadeh A, Amini M, Mansourian M. Predictors of all-cause and cardiovascular-specific mortality in type 2 diabetes: A competing risk modeling of an Iranian population. Adv Biomed Res. 2016;5:82. doi: 10.4103/2277-9175.182213. [PubMed: 27274497]. [PubMed Central: PMC4879860].

16. Drake I, Gullberg B, Sonestedt E, Stocks T, Bjartell A, Wirfalt E, et al. Type 2 diabetes, adiposity and cancer morbidity and mortality risk taking into account competing risk of noncancer deaths in a prospective cohort setting. Int J Cancer. 2017;141(6):1170-80. doi: 10.1002/ijc.30824. [PubMed: 28593629]. [PubMed Central: PMC5575549].

17. Chobanian AV, Bakris GL, Black HR, Cushman WC, Green LA, Izzo JL Jr, et al. Seventh report of the joint national committee on prevention, detection, evaluation, and treatment of high blood pressure. Hypertension. 2003;42(6):1206-52. doi: 10.1161/01.HYP.0000107251.49515.c2. [PubMed: 14656957].

18. Mancia G, Fagard R, Narkiewicz K, Redon J, Zanchetti A, Böhm M, et al. 2013 ESH/ESC Guidelines for the Management of Arterial Hypertension. Blood Press. 2013;22(4):193-278. doi: 10.3109/08037051.2013.812549. [PubMed: 23777479].

19. Austin PC, Lee DS, Fine JP. Introduction to the analysis of survival data in the presence of competing risks. Circulation. 2016;133(6):601-9. doi: 10.1161/CIRCULATIONAHA.115.017719. [PubMed: 26858290]. [PubMed Central: PMC4741409].

20. Dignam JJ, Zhang Q, Kocherginsky M. The use and interpretation of competing risks regression models. Clin Cancer Res. 2012;18(8):23018. doi: 10.1158/1078-0432.CCR-11-2097. [PubMed: 22282466]. [PubMed Central: PMC3328633].

21. Fine JP, Gray RJ. A proportional Hazards model for the subdistribution of a competing risk. J Am Stat Assoc. 1999;94(446):496-509. doi: $10.1080 / 01621459.1999 .10474144$
22. Koller MT, Raatz H, Steyerberg EW, Wolbers M. Competing risks and the clinical community: Irrelevance or ignorance? Stat Med. 2012;31(11-12):1089-97. doi: 10.1002/sim.4384. [PubMed: 21953401]. [PubMed Central: PMC3575691].

23. Gray RJ. A class of $k$-sample tests for comparing the cumulative incidence of a competing risk. Ann Stat. 1988;16(3):1141-54. doi: 10.1214/aos/1176350951.

24. Chen G, McAlister FA, Walker RL, Hemmelgarn BR, Campbell NR. Cardiovascular outcomes in framingham participants with diabetes: The importance of blood pressure. Hypertension. 2011;57(5):891-7. doi: 10.1161/HYPERTENSIONAHA.110.162446. [PubMed: 21403089]. [PubMed Central: PMC3785072].

25. Lu WQ, Resnick HE, Jablonski KA, Jain AK, Jones KL, Robbins DC, et al. Effects of glycaemic control on cardiovascular disease in diabetic American Indians: The strong heart study. Diabet Med. 2004;21(4):3117. doi: 10.1111/j.1464-5491.2004.01137.x. [PubMed:15049931].

26. Bonakdaran S, Ebrahimzadeh S, Noghabi SH. Cardiovascular disease and risk factors in patients with type 2 diabetes mellitus in Mashhad, Islamic Republic of Iran. East Mediterr Health J. 2011;17(9):640-6. doi: 10.26719/2011.17.9.640. [PubMed: 22259913].

27. Sytkowski PA, Kannel WB, D'Agostino RB. Changes in risk factors and the decline in mortality from cardiovascular disease. The framingham heart study. $N$ Engl J Med. 1990;322(23):1635-41. doi: 10.1056/NEJM199006073222304. [PubMed: 2288563]

28. Imperatore G, Cadwell BL, Geiss L, Saadinne JB, Williams DE, Ford ES, et al. Thirty-year trends in cardiovascular risk factor levels among US adults with diabetes: National Health and Nutrition Examination Surveys, 1971-2000. Am J Epidemiol. 2004;160(6):531-9. doi: 10.1093/aje/kwh232. [PubMed: 15353413].

29. LaRosa JC. Understanding risk in hypercholesterolemia. Clin Cardiol. 2003;26(1 Suppl 1):I3-6. doi: 10.1002/clc.4960261303. [PubMed: 12539815].

30. Jousilahti P, Vartiainen E, Pekkanen J, Tuomilehto J, Sundvall J, Puska P. Serum cholesterol distribution and coronary heart disease risk: Observations and predictions among middle-aged population in eastern Finland. Circulation. 1998;97(11):1087-94. [PubMed: 9531256].

31. Cho E, Rimm EB, Stampfer MJ, Willett WC, Hu FB. The impact of diabetes mellitus and prior myocardial infarction on mortality from all causes and from coronary heart disease in men. $J$ Am Coll Cardiol. 2002;40(5):954-60. doi: 10.1016/S0735-1097(02)02044-2. [PubMed: 12225722].

32. Wannamethee SG, Shaper AG, Whincup PH, Lennon L, Sattar N. Impact of diabetes on cardiovascular disease risk and all-cause mortality in older men: Influence of age at onset, diabetes duration, and established and novel risk factors. Arch Intern Med. 2011;171(5):404-10. doi: 10.1001/archinternmed.2011.2. [PubMed: 21403036].

33. Brun E, Nelson RG, Bennett PH, Imperatore G, Zoppini G, Verlato G, et al. Diabetes duration and cause-specific mortality in the Verona diabetes study. Diabetes Care. 2000;23(8):1119-23. doi: 10.2337/diacare.23.8.1119. [PubMed: 10937508].

34. Boden-Albala B, Cammack S, Chong J, Wang C, Wright C, Rundek T, et al. Diabetes, fasting glucose levels, and risk of ischemic stroke and vascular events: Findings from the northern manhattan study (NOMAS). Diabetes Care. 2008;31(6):1132-7. doi: 10.2337/dc07-0797. [PubMed: 18339972].

35. Lehto S, Ronnemaa T, Pyorala K, Laakso M. Predictors of stroke in middle-aged patients with non-insulin-dependent diabetes. Stroke. 1996;27(1):63-8. doi: 10.1161/01.STR.27.1.63. [PubMed: 8553405].

36. Janghorbani M, Hu FB, Willett WC, Li TY, Manson JE, Logroscino G, et al. Prospective study of type 1 and type 2 diabetes and risk of stroke subtypes: The nurses' health study. Diabetes Care. 2007;30(7):1730-5. doi: 10.2337/dc06-2363. [PubMed: 17389335].

37. Banerjee C, Moon YP, Paik MC, Rundek T, Mora-McLaughlin C, Vieira JR, et al. Duration of diabetes and risk of ischemic stroke: The northern manhattan Study. Stroke. 2012;43(5):1212-7. doi: 
10.1161/STROKEAHA.111.641381. [PubMed: 22382158]. [PubMed Central: PMC3336044].

38. Malek M, Aghili R, Emami Z, Khamseh ME. Risk of cancer in diabetes: The effect of metformin. ISRN Endocrinol. 2013;2013:636927. doi: 10.1155/2013/636927. [PubMed: 24224094]. [PubMed Central: PMC3800579].

39. Vupputuri S, Nichols GA, Lau H, Joski P, Thorp ML. Risk of progression of nephropathy in a population-based sample with type 2 diabetes. Diabetes Res Clin Pract. 2011;91(2):246-52. doi: 10.1016/j.diabres.2010.11.022. [PubMed: 21156326].

40. Saberi H, Mohammadtaghvaei N, Gulkho S, Bakhtiyari S, Moham- madi M, Hanachi P, et al. The ENPP1 K121Q polymorphism is not associated with type 2 diabetes and related metabolic traits in an Iranian population. Mol Cell Biochem. 2011;350(1-2):113-8. doi: 10.1007/s11010010-0687-z. [PubMed: 21153685].

41. Levin ME. Foot lesions in patients with diabetes mellitus. Endocrinol Metab Clin North Am. 1996;25(2):447-62. doi: 10.1016/s08898529(05)70333-9.

42. Walters DP, Gatling W, Mullee MA, Hill RD. The distribution and severity of diabetic foot disease: A community study with comparison to a non-diabetic group. Diabet Med. 1992;9(4):354-8. doi: 10.1111/j.14645491.1992.tb01796.x. [PubMed: 1600707]. 